CULTURA, LENGUAJE Y REPRESENTACIÓN / CULTURE, LANGUAGE AND REPRESENTATION · ISSN 1697-7750 • VOL. XV \2016, PP. 51-63 REVISTA DE ESTUDIOS CULTURALES DE LA UNIVERSITAT JAUME I / CULTURAL STUDIES JOURNAL OF UNIVERSITAT JAUME I

DOI: HTTP://DX.DOI.ORG/10.6035/CLR.2016.15.4

\title{
La nueva narrativa identitaria del populismo: un análisis del discurso de Pablo Iglesias (Podemos) en Twitter
}

\author{
The new identitary narrative of populism: A discourse analysis \\ of Pablo Iglesias (Podemos) on Twitter
}

ENRIQUE ARROYAS LANGA

PEDRO LUIS PÉREZ DÍAZ

UNIVERSIDAD CATÓLICA SAN ANTONIO DE MURCIA

Recibido: 10-10-2014

Aceptado: 01-03-2015

RESUMEN: El término «populista», que ha sido utilizado para identificar de forma peyorativa determinadas prácticas demagógicas tanto de la izquierda como de la derecha, ha cobrado protagonismo en los últimos tiempos en un contexto de crisis de representación de las democracias, desafección política de la ciudadanía y pérdida de confianza en las instituciones. No obstante, también se ha abordado este fenómeno político sin connotaciones negativas, de forma que pueda estudiarse el populismo como un discurso político presente en cualquier democracia. Siguiendo este enfoque teórico, en el análisis que aquí se presenta no se aborda el populismo como una doctrina, sino como un estilo discursivo que se basa en el enfrentamiento entre el pueblo y el poder (Laclau, 2005), la construcción de un líder fuerte y la crítica hacia las estructuras tradicionales de la política. El éxito electoral y comunicativo de la formación Podemos tras las elecciones al Parlamento Europeo de 2014 lo convierte en un ejemplo significativo de nuevas formas de hacer política que aprovecha las redes sociales de Internet para construir relaciones con la sociedad civil y difundir sus mensajes. Bajo este pretexto, la presente investigación analiza los elementos populistas de la estrategia discursiva en Twitter del líder de Podemos, Pablo Iglesias Turrión (@Pablo_Iglesias_),durante la campaña de las mencionadas elecciones europeas. Para ello, a partir del trabajo de Charaudeau (2009), se realiza un análisis de contenido de los 249 tuits que conformaron su discurso en este periodo, con el objetivo de revelar si se ajusta a los parámetros del estilo populista. En los resultados se han apreciado similitudes con las estrategias discursivas del populismo: una narrativa cuyo motor es la confrontación entre el ellos y el nosotros, la identificación abstracta y sin matices del adversario y la exaltación de un ideal de comportamiento honesto frente a la corrupción. No obstante, el estilo populista de Podemos aparece 
matizado por la presentación de un líder cuyos atributos refuerzan su conexión con la ciudadanía más que su carisma individual.

Palabras clave: populismo, discurso, comunicación política, Twitter, Podemos.

ABSTRACT: The term "populist», which has been used pejoratively to identify certain demagogic practices from both the left and the right, has gained prominence in recent years amid a global economic crisis that has deepened the crisis of representative democracies, political disaffection and loss of confidence in institutions. However, this political phenomenon has also been addressed without negative connotations, so that populism can be studied as a political discourse present in any democracy since the late twentieth century. Following this theoretical approach, in the analysis presented here populism isn't treated as a doctrine but as a discursive style that is based on the confrontation between the people and power (Laclau, 2005), the building of a strong leader and the criticism of the traditional structures of politics. The electoral and communicative success of Podemos after the European Parliament election of 2014 in Spain turned it a significant example of these new ways of doing politics that leverages social networking websites to build relationships with civil society and spread their messages. Under this pretext, this research analyzes the populist elements in the discursive strategy of Podemos leader, Pablo Iglesias Turrión (@Pablo_Iglesias_), during the campaign of the aforementioned European elections. To accomplish this, basing our analysis on the work of Charaudeau (2009), we make a content analysis of 249 tweets that shaped his speech in this period, with the aim of revealing whether it adapts to the parameters of populist style. The results show similarities to the discursive strategies of populism: a narrative whose engine is confrontation between them and us, the abstract and blurry identification of the adversary and the exaltation of an ideal of honest behavior against corruption. However, the populist style of Podemos appears tinged with the presentation of a leader whose attributes reinforce their connection with citizenship rather than their individual charisma.

Keywords: populism, discourse, political, communication, Twitter, Podemos.

\section{Introducción}

En un reportaje sobre Podemos publicado en El País, John Carlin (2015) sostiene que la formación de Pablo Iglesias tiene algo que cualquier partido necesita para ganar unas elecciones, una narrativa identitaria asumible por una mayoría amplia de la población: «se presentan al imaginario colectivo como los caballeros de la Mesa Redonda que, junto al pueblo enardecido, pretenden 
atacar, despoblar y ocupar el castillo negro donde se atrinchera la despiadada casta».

Para la configuración de esta narrativa, Podemos ha recurrido a la antítesis de dos fuerzas en un momento de desencanto con la democracia y de aumento de las desigualdades: la gente frente al poder, la ilusión de cambio de la mayoría frente al inmovilismo de los grandes partidos, considerados como una casta que defiende un statu quo basado en los privilegios y la corrupción. Esta batalla de ideas se presenta, además, como crucial para el futuro del país y novedosa porque de ella surgirá una democracia mejor gracias a una nueva forma de hacer política que, representada por Podemos, rompe con la vieja dicotomía derecha-izquierda, que ya no sirve cuando lo urgente es devolver a la gente el poder que está secuestrado por la elite oligárquica.

El fenómeno de Podemos, que ha irrumpido con fuerza en el debate político español, se inserta en una situación de crisis de la representación política y en medio de una crisis económica mundial con efectos graves sobre la ciudadanía. Esta doble crisis ha puesto de manifiesto una situación social convulsa, en la que se percibe un aumento de las desigualdades y una serie de disfunciones en el sistema político cuya consecuencia más palpable es una creciente desconfianza de los ciudadanos hacia los políticos.

Esta desafección no sería tanto un desinterés del ciudadano hacia la política como una desconfianza hacia el sistema. Lo característico del espacio público en esta etapa de postpolítica sería una combinación de rechazo de las formas clásicas de organización con un incremento del compromiso individual a través de nuevas formas de activismo y movilización ciudadana (Innerarity, 2014).

En este contexto, Podemos recogió el testigo de las movilizaciones del $15 \mathrm{M}$ y a la reclamación de un saneamiento de la democracia añadió la voluntad de ofrecer una nueva forma de hacer política, que incluía, además de la promesa de honradez, la ruptura de la brecha entre gobernantes y gobernados mediante la devolución del poder de decisión a los ciudadanos reunidos en asambleas deliberativas. Se presentaban de este modo como una recuperación de la auténtica política o como antídotos de la antipolítica, consiguiendo de esta forma aparecer como la solución al problema más acuciante del momento: la doble crisis política y económica: «este es uno los antídotos que partidos y líderes políticos utilizan para protegerse de la antipolítica. Motivar a los electores enalteciendo las virtudes del pueblo y denunciando las elites corruptas y depredadoras que causan las adversidades de la sufrida nación es una estrategia muy antigua» (Naím, 2014).

Como fuerza que surge desde los movimientos sociales externos al sistema político y que aspira a reunir apoyos para la conquista del poder, en su estrate- 
gia el discurso juega un papel fundamental, pues será a través de la construcción de una narrativa simbólica el medio con el que lograrán ser percibidos como la alternativa. La caracterización de un discurso político como populista en un contexto de deliberación democrática requiere una previa clarificación conceptual del término.

\section{Una aproximación al concepto de «populismo»}

El populismo ha sido un fenómeno renuente a ser definido con precisión, pues ha servido para definir movimientos políticos, partidos y líderes en una amplia variedad de contextos geográficos, históricos e ideológicos. Tras una revisión de la literatura especializada, (Canovan, 1981; Taggart, 2000) abordaremos el concepto «populismo» desde tres perspectivas (Weyland, 2001; Hurtado, 2011; Gidron y Bonikowski, 2013): como ideología, como estilo discursivo y como estrategia politica.

Como ideología política, se trata de una «levemente centrada (thin-centered ideology) que considera que la sociedad está en última instancia separada en dos grupos homogéneos y antagónicos, 'el puro pueblo' frente a 'la corrupta elite', que defiende que la política debe ser una expresión de la voluntad general de las personas» (Mudde, 2004: 543).

Esta primera aproximación, el populismo como conjunto de ideas, conduce a la segunda: un estilo discursivo que encaja el debate público en el marco de una retórica maniquea y moralmente binaria. Desde este punto de vista, el populismo debe ser estudiado en su dimensión expresiva y creadora de identidades políticas.

La tercera corriente ubica al populismo en el ámbito de la estrategia política, como un modo de ejercer el poder. El líder populista se muestra cercano al «pueblo» mediante un proceso de identificación en el que las movilizaciones de masas y la permanente presencia en los medios de comunicación sirve para recordar a las mayorías populares que él conoce y experimenta las mismas dificultades que el resto, al tiempo que promete una transformación en el espacio político: los intereses particulares de la oligarquía serán sustituidos por el interés general.

Los defensores de la acepción ideológica del concepto entienden que, por abstracta, la aproximación discursiva es demasiado teórica y asemeja el populismo a cualquier formulación de retórica dualista -el binomio puebloélite puede ser sustituido sin dificultad por cualquier nosotros-ellos- (Gidron y Bonikowski, 2013). Por su parte, quienes defienden la visión discursiva no 
admiten la limitación que supone igualar el populismo a la ideología, puesto que convienen que en realidad las formas populistas están disponibles para cualquier actor político que las puede emplear a conveniencia como un modo flexible de persuasión para redefinir a la gente y a sus adversarios (Panizza, 2005: 8).

De hecho, las dúctiles formas populistas han barnizado comportamientos de todo signo político: caudillos latinoamericanos del primer tercio del siglo XX como Perón en Argentina, neopopulistas de derechas de los ochenta como el peruano Fujimori, políticos europeos con rasgos xenófobos como el austriaco Haider y el francés Le Pen, líderes aupados por imperios mediáticos como Berlusconi y los más recientes desde el ámbito de la izquierda antiimperialista como Chávez en Venezuela o Morales en Bolivia (Hurtado, 2011: 27).

Desde posiciones filosóficas liberales se ha subrayado la manifiesta incompatibilidad entre populismo y democracia: el populismo no sería más que una forma degenerada de democracia. No en vano, la génesis del fenómeno se produce en las cíclicas crisis de descontento de los estados liberales democráticos: solo la democracia puede conferirle el grado de libertad que requiere para nacer y desarrollarse (Hernández y Hurtado, 2010).

La visión peyorativa del fenómeno ha sido ampliamente discutida por el marxismo postestructuralista, donde adquiere protagonismo el pensamiento del teórico político argentino Ernesto Laclau, uno de los principales responsables de rescatar este concepto de los márgenes del debate intelectual. En su obra $L a$ razón populista (2005), Laclau niega toda categoría ideológica al populismo, definiéndolo más como una forma discursiva de crear identidades políticas que siempre se construyen antagónicamente, pues la democracia es un campo de permanente conflicto social.

Laclau concede que los populismos hacen un uso habitual de la retórica y atribuye a esta un rol fundamental en la dimensión conflictiva de la democracia, ya que son operaciones de naturaleza retórica las que constituyen al sujeto político y definen coyunturalmente las relaciones entre éste, otros sujetos y el poder. Sus ideas sobre la hegemonía, atravesadas por el psicoanalismo de Lacan, se fundamentan en el siguiente planteamiento: «la multitud de significantes flotantes [...] está estructurada en un campo unificado a través de la intervención de un determinado punto nodal (el point de capiton lacaniano) que los acolcha» (Žižek, 1992: 125), dentro del cual «el discurso se constituye como intento por dominar el campo de la discursividad, por detener el flujo de las diferencias, por constituir un centro» (Laclau y Mouffe, 2004: 152).

Según esta perspectiva, el discurso populista se elabora en torno a multitud de significantes «vacíos» o «flotantes» que se deslizan a la espera de ser 
colmados con un significado que fije su sentido a través de un punto nodal que acolche a esos significantes en una cadena de equivalencias, un plus metafórico (Žižek, 1992) que los conecte y los identifique de un modo totalizador. Por tanto, lo político no sería más que una pugna constante por conquistar estos significantes abiertos para engarzarlos en un sentido legitimador. Por ejemplo, los significantes vacíos «paro», «corrupción», «privilegios» pueden ser acolchados al punto nodal «casta», de modo que ese anudamiento se vuelva hegemónico. El nuevo relato populista se extiende e invade el lenguaje cotidiano, estableciendo un nuevo tablero de juego con unas nuevas reglas que todos los jugadores políticos terminan por aceptar.

El propio Íñigo Errejón, jefe de campaña de Podemos durante las elecciones al Parlamento Europeo de 2014, recurrió frecuentemente a Laclau en su tesis doctoral, donde define el populismo como «un estilo discursivo que unifica simbólicamente posiciones muy diferentes y muy fragmentadas en una simplificación del espacio político, que lo dicotomiza, dividiendo entre el pueblo como representante de la voluntad general y las elites» (ATTAC.TV, 2012).

La interpelación al pueblo es habitualmente realizada por un líder carismático fuerte, carente de un programa político y comprometido con romper con las prácticas políticas del pasado, acabar con la corrupción y empoderar a la soberanía popular (Charaudeau, 2009: 260). Simbólicamente, la individualidad del líder congrega las heterogéneas aspiraciones que ahora se polarizan alrededor de una identidad homogénea - pueblo- en contra de una elite que instrumentaliza el poder en beneficio propio. En este proceso, el caudillaje del líder es, según Laclau, indispensable: no he encontrado ningún caso histórico en que esta reconstitución de la identidad nacional ocurra sin la personalidad ni la figura de un líder (Muñoz, 2014).

Por ello, partimos de la premisa planteada por Gerbaudo (2015) de que el hombre común (trabajador, víctima del sistema político y financiero) al que tradicionalmente ha apelado la narrativa populista encuentra su actualización en el «usuario genérico de Internet» que, con tan solo una conexión a la red y con indiferencia de otros rasgos sociodemográficos, se convierte en un potencial participante de la arena política y es susceptible de ser sujeto integrado en la colectividad, en el pueblo.

\section{Metodología y caso de estudio}

Para el análisis del discurso construido por el líder de Podemos, Pablo Iglesias Turrión (@Pablo_Iglesias_), en Twitter se recogieron todos los tuits 
que el líder de la formación publicó en esta red social durante la campaña electoral (del 9 al 25 de mayo de 2014). Para ello, se empleó la herramienta YourTwapperKeeper, que archivó un total de 249 tuits en el mencionado periodo de estudio y cuya explotación estadística se llevó a cabo en SPSS 20.

El análisis de contenido cuantitativo y cualitativo de este corpus textual se realiza a partir de las categorías establecidas por Charaudeau (2009), para quien el discurso populista basa su fuerza persuasiva en la escenificación de una situación social catastrófica, la identificación de una elite culpable, la exaltación de valores de gran carga simbólica y la aparición de una alternativa salvadora.

A partir de este planteamiento teórico, sintetizamos la hoja de codificación expuesta en la tabla 1, que revela los rasgos definitorios de la imagen de Pablo Iglesias como líder y la construcción de la narrativa simbólica que ofrece el proyecto de Podemos. El análisis se ha centrado en los aspectos nucleares del discurso relacionados con las ideas que se defienden y los valores que las respaldan, mientras que los aspectos lingüístico-formales serán analizados en etapas posteriores de la presente investigación.

\section{Tabla 1. Hoja de codificación empleada en el análisis}

\section{Tema}

- Movilización: apelaciones directas a la acción popular para la consecución del cambio.

- Crisis económica: este tema está enfocado hacia la desigualdad entre ricos y pobres.

- Decadencia moral: causada por la pérdida de un sentido cívico de la política.

- Victimización de los ciudadanos: quienes sufren las consecuencias de un poder injusto.

\section{Historia}

Se trata de desvelar la narrativa que construye el discurso para explicar la situación de crisis política. Para ello, se realiza la identificación de los personajes que intervienen en el discurso. En primer lugar, se identifica al culpable de los males del país con términos abstractos. Cuando en ese discurso se personaliza al culpable se hace a modo de recurso metonímico, con el que la persona señalada aparece como chivo expiatorio. Las categorías analizadas son:

- Ellos

- Nosotros

- Ellos contra nosotros 


\section{Valores}

Se exaltan valores con fuerza simbólica que remiten a un ideal de comportamiento que contrasta con la corrupción imperante: honestidad, nobleza, autenticidad, pureza. En este aspecto, los valores sirven para sustentar toda la narrativa populista de antítesis. Las categorías de análisis que hemos establecido son las siguientes:

- Fuerza y unidad (valor relacionado con la movilización popular).

- Solidaridad con el hombre común (valor que persigue la adhesión a los sectores más desprotegidos y que se identifica con la gente corriente).

- Honestidad (valor relacionado con la transparencia, la nobleza, la pureza, etc.).

- Diferencia (valor relacionado con la disimilitud, el 'nosotros' se construye por contraposición al 'ellos').

- Cambio (valor que prioriza la ruptura como alternativa al inmovilismo institucional).

\section{Atributos del líder ${ }^{1}$}

La fuerza movilizadora del discurso populista se sirve de la construcción de un personaje protagonista que actúa como símbolo de los valores con los que se identificará el pueblo.

1. Benevolencia

- Honradez

- Capacidad de llegada, cercanía

- Empatía

\section{Prudencia}

- Coherencia

- Responsabilidad

- Equipo

- Oratoria

- Presencia física

3. Virtud

- Dinamismo

- Competencia

- Eficacia

- Experiencia política

Fuente: elaboración propia a partir de Charaudeau (2009)

1. Los atributos que conforman el rasgo principal del ethos se detallan en un estado previo de este aspecto en la figura de Pablo Iglesias, véase Pérez Díaz, Martínez y Correyero (2014). 


\section{Análisis de resultados}

En cuanto a los temas y las tesis defendidas, destaca en primer lugar la movilización popular. Así, la mitad de los tuits se refieren a la organización de actos de campaña, lo que se antoja lógico teniendo en cuenta las fechas elegidas para el análisis. Lo peculiar en cuanto al contenido es el enfoque de estos escritos: la campaña se entiende como un esfuerzo colectivo a pie de calle. Esta forma de entender la campaña es el primer paso para la construcción de esa narrativa que hace Podemos para dar sentido a la realidad social del país como una confrontación entre ciudadanía y poder establecido.

A continuación, con un 30,9\%, el tema que predomina es la decadencia moral por la pérdida de un sentido cívico de la política. Este tema engloba la denuncia de la corrupción, el anquilosamiento de los partidos y los privilegios de los políticos. El resto de tuits se reparten entre denuncias de la desigualdad entre ricos y pobres $(4,4 \%)$ y el señalamiento de las consecuencias de la crisis en forma de sufrimiento para los ciudadanos (6\%).

El análisis de la trama narrativa arroja algunos resultados interesantes en cuanto a las estrategias del estilo populista. Lo que predomina es la configuración del nosotros vinculada al relato de la movilización popular como el comienzo esperanzador de un cambio. El 49,4\% de los tuits hablan de ese compromiso colectivo en cada ciudad visitada por Iglesias como parte de la caravana electoral. Esta característica de la narrativa de Podemos sería algo específico del discurso populista que se explica por un contexto en el que la crisis está tan consolidada y asumida por el imaginario colectivo que no es necesario dedicar un gran esfuerzo persuasivo para convencer al auditorio de la gravedad de la situación.

Como es propio del discurso populista, la identificación del adversario se hace de forma abstracta y sin matices (12,9\%). El enemigo suele ser identificado como «la casta» o «los banqueros», aunque en algunas ocasiones se personaliza al culpable señalándolo como chivo expiatorio. Frente a ellos, el nosotros se asocia a términos positivos como «transparencia», «ilusión»o «esperanza».

Los tuits en los que se menciona la confrontación alcanzan el 23,7 \%. Un ejemplo lo tenemos en el siguiente tuit: «Blesa se va a Londres y el hotel se lo pagamos todos. Diplomacia para ricos, cunetas para pobres», que sirve como modelo de la narrativa de Podemos, cuyo argumento se resume en una situación de decadencia, causada por la corrupción de la elite, cuya consecuencia es la desigualdad y el sufrimiento de la gente, y que exige de forma urgente un cambio drástico que ahora es posible gracias a la movilización popular liderada 
por un partido que, gracias a una nueva forma de hacer política, cuenta con la fuerza del apoyo de la ciudadanía conquistada por su honestidad.

La antítesis entre el ellos y el nosotros, junto a la demonización del contrincante, es la base de la fuerza persuasiva de la narrativa de Podemos cuando el mensaje toma forma con la construcción de personajes prototípicos como el héroe y el villano. En este aspecto, es muy significativo que la presentación del líder como héroe aparezca siempre matizada por el respaldo del grupo, sea su equipo de campaña (muy destacados en el papel de organizadores de los actos a pie de calle), sea la ciudadanía movilizada. En los tuits referidos al héroe, siempre encontraremos la primera persona del plural: «Esta campaña es diferente porque la hacemos entre todos. Es nuestro momento», «logremos hacer algo grande juntos». Esta estrategia de construcción del nosotros como héroe colectivo matiza el estilo populista clásico que, aunque también busca la conexión con el sentir popular, se basa en el carisma del líder.

Los resultados del análisis respaldan la escala de valores habitual del discurso populista basado en la exaltación de un ideal de comportamiento honesto por contraste con la corrupción imperante. Las promesas de cambio y la fuerza de la movilización predominan por encima de las soluciones concretas $(46,1 \%)$. Y se puede destacar la coherencia del discurso, en el que todos los valores están relacionados unos con otros y vinculados con la narrativa esencial de Podemos trenzada en la antítesis nosotros/ellos, lo que le da fuerza persuasiva.

Junto a la fuerza de la unidad (36,9\%), el valor principal que defienden es la honestidad frente a la corrupción política, junto a la transparencia frente a la opacidad de las complicidades actuales entre partidos y bancos $(25,3 \%)$ : «\#Yo voto podemos porque no hemos pedido créditos a los bancos, porque nuestras cuentas son transparentes y nos financia la gente». Estos valores son los que les hacen diferentes y, por lo tanto, susceptibles de propiciar el cambio (9,2\%): «33.000 votantes en las primarias, 400 círculos, cuatro meses en los que hemos pasado de no existir a ser algo imposible de ignorar».

«Somos independientes porque la fuerza de Podemos es la ilusión y el trabajo de mucha gente y no el dinero de la banca. \#SinBancosPodemos». Este tuit sintetiza la jerarquía de valores que sostiene la narrativa de Podemos. La honestidad y la transparencia como valores nucleares que les hacen diferentes a la casta y, a través de su solidaridad con la gente que más sufre la crisis, les ha permitido conseguir un fuerte respaldo popular por la ilusión despertada para el cambio.

Para completar esta narrativa identitaria, el rasgo constitutivo del ethos que predominó en la imagen de Pablo Iglesias se basó en una combinación de benevolencia, prudencia y virtud, con predominio de la primera. Tres atributos 
configuran su liderazgo: la honradez, que le hace revestirse de una apariencia radicalmente diferente de la elite corrupta; la empatía, que le permite actuar como reflejo de un gran sector de ciudadanos desencantados con la política; y el dinamismo, con el que trata de identificar a Podemos con un movimiento impulsado por la unidad popular y con fuerza para ganar las elecciones.

\section{Conclusiones}

Lo primero que se aprecia en el análisis de resultados es una gran coherencia entre los cuatro apartados analizados en la construcción del discurso de Pablo Iglesias en Twitter. Entre los temas abordados (la narrativa explicativa, los valores que se defienden y los atributos que configuran la imagen del líder de Podemos) existe una coherencia que, sin duda, es una de las razones de su fuerza persuasiva. En todos ellos encontramos similitudes con las estrategias discursivas del estilo populista.

El gran tema del discurso de Iglesias, y por lo tanto aquello con lo que se identifica como su prioridad, es la fuerza de la movilización popular en un momento crucial de decadencia moral o deterioro de la democracia que Podemos ha venido a cambiar. Este tema se desarrolla con una narrativa cuyo motor es la confrontación entre el ellos y el nosotros, entre la casta corrupta y Podemos como aglutinador de un apoyo popular que posee fuerza e ilusión. Como es propio del discurso populista, la identificación del adversario se hace de forma abstracta y sin matices o personalizada en un chivo expiatorio. La escala de valores es la habitual del discurso populista, basado en la exaltación de un ideal de comportamiento honesto y transparente por contraste con la corrupción imperante y capaz de conectar con la gente corriente.

El aspecto que podríamos identificar como específico de Podemos y menos asimilable al estilo populista es la presentación del líder, ya que en lugar de apostar por el carisma individual capaz de arrastrar a las masas, aparece siempre matizado por el respaldo del grupo, creándose así una imagen de héroe colectivo.

A la luz de este análisis, se puede concluir que el discurso del líder de Podemos reflejado en Twitter comparte con el estilo populista la narrativa basada en la denuncia de una elite política y financiera a la que se señala, sin matices, como culpable de la decadencia de la democracia, y su contraposición con la alternativa nueva, honesta y transparente simbolizada por Podemos, que recoge el malestar expresado por un amplio sector de la población y, gracias 
a una movilización popular, ofrece un cambio que se visualiza como crucial en una situación de crisis.

\section{Referencias bibliográficas}

AtTac.TV (2012): Íñigo Errejón - Populismo, reforma y transición, 23 de febrero, vídeo recuperado de: http://vimeo.com/37029639.

Canovan, M. (1981): Populism, Nueva York, Harcourt Brace Jovanovich.

Carlin, J. (2015): Los caballeros de la Mesa Redonda. El País, 27 de enero, recuperado de: http://politica.elpais.com/politica/2015/01/27/ actualidad/1422384264_753104.html.

Charaudeau, P. (2009): «Reflexiones para el análisis del discurso populista», Discurso y Sociedad, 3 (2): 253-279, recuperado de: http://www.dissoc. org/ediciones/v03n02/DS3\%282\%29Charaudeau.pdf.

ERReJón, I. (2012): La lucha por la hegemonía durante el primer gobierno del MAS en Bolivia (2006-2009): un análisis discursivo (tesis doctoral), Universidad Complutense de Madrid, Madrid, recuperada de: http://eprints. ucm.es/14574/1/T33089.pdf.

Gerbaudo, P. (2015): «Populism 2.0. Social Media Activism, the generic Internet user and interactive direct democracy», en Trottier, D.; C. Fuchs (eds.): Social Media, Politics and the State: Protests, Revolutions, Riots, Crime and Policing in the Age of Facebook, Twitter and Youtube, Nueva York, Routledge, 67-88.

Gidron, N.; B. Bonikowski (2013): Varieties of Populism: Literature Review and Research Agenda, Weatherhead Working Paper Series, No. 130004, recuperado de: http://scholar.harvard.edu/files/gidron_bonikowski_populismlitreview_2013.pdf.

Hernández, I.; H. Hurtado (2010): El populismo. Una aproximación a un problema de las democracias de todos los tiempos, San José (Costa Rica), Facultad Latinoamericana de Ciencias Sociales (FLACSO), recuperado de: http://www.academia.edu/1524312/El_populismo._Una_ aproximaci\%C3\%B3n_a_un_problema_de_las_democracias_de_todos_ los_tiempos.

Hurtado, H. (2011): "Aproximación a un concepto "esencial" de populismo», Temas de coyuntura, diciembre de 2010, 62: 11-36, recuperado de: http://www.academia.edu/1524288/Aproximaci\%C3\%B3n_a_un_concepto_esencial_del_populismo. 
INNERARITY, D. (2014): «Democracia sin política», El País, 28 de febrero, recuperadode: http://elpais.com/elpais/2014/02/19/opinion/1392837582_448839. html.

LACLAU, E. (2005): La razón populista, Buenos Aires, Fondo de Cultura Económica.

LaClau, E.; C. Mouffe (2004): Hegemonía y estrategia socialista, Buenos Aires, Fondo de Cultura Económica.

March, L. (2007): «From Vanguard of the Proletariat to Vox Populi: LeftPopulism as a 'Shadow' of Contemporary Socialism», SAIS Review, 27 (1): 63-77.

Mudde, C. (2004): «The Populist Zeitgeist», Government \& Opposition, 39 (4): 541-563.

MuÑoz, B. (2014): Entrevista a Ernesto Laclau [1935-2014] «Todo populismo es un momento de ruptura», Prodavinci.com, 14 de abril, recuperado de: http://prodavinci.com/2014/04/14/actualidad/ernesto-laclau$\%$ E2\%80\%9Ctodo-populismo-es-un-momento-de-ruptura $\% \mathrm{E} 2 \% 80 \% 9 \mathrm{D} /$.

Naím, M. (2014): «¿Qué le pasa a la política?», El País, 11 de octubre, recuperado de: http://internacional.elpais.com/internacional/2014/10/11/ actualidad/1413056690_149449.html.

Panizza, F. (2005): Populism and the Mirror of Democracy, Londres, Verso.

Pérez díaz, P. L.; H. Martínez Martínez; B. Correyero Ruiz (en prensa): «Twitter y retórica: el ethos como recurso persuasivo en el caso de Pablo Iglesias (Podemos)», capítulo de un libro de próxima publicación en Ediciones Universitarias de McGraw-Hill.

Taggart, P. (2000): Populism, Buckingham, Open University Press.

Weyland, K. (2001): «Clarifying a Contested Concept: Populism in the Study of Latin American Politics», Comparative Politics, 34 (1): 1-22.

ŽıžEK, S. (1992): El sublime objeto de la ideología, México, Siglo XXI. 